\title{
Smart Antennas for Mobile Communications
}

\author{
Bakshi Bhagyashri Renukadas \\ (Electronics and communication Department, G.P.Beed,India)
}

\begin{abstract}
This paper presents brief account on smart antenna (SA) systemfor mobile wireless communications, The adoption of smart / adaptive antenna techniques in future wireless systems is expected to have a significant impact on the efficient use of the spectrum, the minimization of the cost of establishing new wireless networks, the optimization of service quality and realization of transparent operation across multi technology wireless networks [1]. This paper presents brief account on smart antenna (SA) system. SAs can place nulls in the direction of interferers via adaptive updating of weights linked to each antenna element. SAs thus cancel out most of the co-channel interference resulting in better quality of reception and lower dropped calls. The paper further explains about the radiation pattern of the antenna and why it is highly preferred in its relative field. The capabilities of smart / adaptive antenna are easily employable to Cognitive Radio and OFDMA system.

Keywords: Smart / Adaptive Antenna; Wireless; Beam forming; DSP; Diversity.
\end{abstract}

\section{Introduction}

In view of explosive growth in the number of digital cellular subscribers, service providers are becoming increasingly concerned with the limited capacities of their existing networks. This concern has led to the deployment of smart antenna systems throughout major metropolitan cellular markets A smart antenna is an array of elements connected to a digital signal processor. Such a configuration dramatically enhances the capacity of a wireless link through a combination of diversity gain, array gain, and interference suppression. Increased capacity translates to higher data rates for a given number of users or more users for a given data rate per user. Multipath paths of propagation are created by reflections and scattering. Also, interference signals such as that produced by the microwave oven in the picture, are superimposed on the desired signals. Measurements suggest that each path is really a bundle or cluster of paths, resulting from surface roughness or irregularities. The random gain of the bundle is called Multipath fading.

This paper mainly concentrates on use of smart antennas in mobile communications that enhances the capabilities of the mobile and cellular system such as faster bit rate, multi-use interference, space division multiplexing (SDMA), adaptive SDMA [21], increase in range, multipath mitigation, reduction of errors due to multipath fading, best suitability of multi-carrier modulations such as OFDMA The advantage of SAs application in cellular systems are decreased inter symbol interference, decreased co-channel interference \& adjacent channel interference, improved bit error rate (due to decreased amount of multipath and ISI), increase in receiver sensitivity, reduction in power consumption \& RF pollution. Smart antennas are most appropriate for use of cognitive radio (software radio technology provides flexibility) and the greatest advantage of smart antenna is a very high security.

\subsection{Smart}

\section{Smart Antenna}

The concept of using multiple antennas and innovative signal processing to serve cells more intelligently has existed for many years. In fact, varying degrees of relatively costly smart antenna systems have already been applied in defense systems. Until recent years, cost barriers have prevented their use in commercial systems. The advent of powerful low-cost digital signal processors (DSPs), general-purpose processors (and ASICs), as well as innovative software-based signal-processing techniques (algorithms) have made intelligent antennas practical for cellular communications systems.

This system is providing greater coverage area for each cell site, higher rejection of interference and substantial capacity improvements.

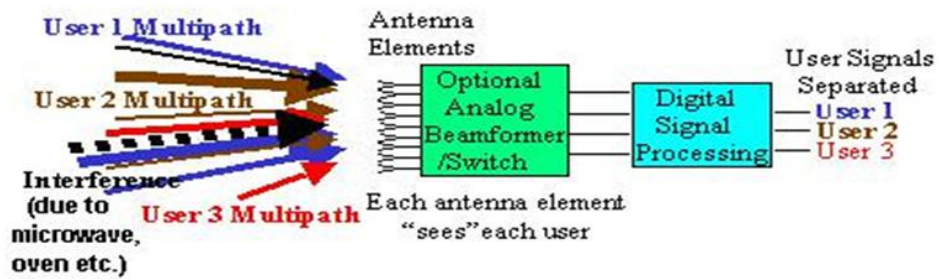

Fig. 1: Smart Antenna System 


\subsection{Principal of Smart Antenna System}

The smart antenna works as follows. Each antenna element "sees" each propagation path differently, enabling the collection of elements to distinguish individual paths to within a certain resolution. As a consequence, smart antenna transmitters can encode independent streams of data onto different paths or linear combinations of paths, thereby increasing the data rate, or they can encode data redundantly onto paths that fade independently to protect the receiver from catastrophic signal fades, thereby providing diversity gain. A smart antenna receiver can decode the data from a smart antenna transmitter this is the highest-performing configuration or it can simply provide array gain or diversity gain to the desired signals transmitted from conventional transmitters and suppress the interference [1]. No manual placement of antennas is required. The smart antenna electronically adapts to the environment.

In truth, antennas are not smart antenna systems are smart. Generally co-located with a base station, a smart antenna system combines an antenna array with a digital signal-processing capability to transmit and receive in an adaptive, spatially sensitive manner. Such a configuration dramatically enhances the capacity of a wireless link through a combination of diversity gain, array gain and interference suppression. Increased capacity translates to higher data rates for a given number of users or more users for a given data rate per user. In other words, such a system can automatically change the directionality of its radiation patterns in response to its signal environment. This can dramatically increase the performance characteristics (such as capacity) of a wireless system.

Multipath of propagation are created by reflections and scattering. Also, interference signals such as that produced by the microwave oven in the picture fig (1) are superimposed on the desired signals. Measurements suggest that each path is really a bundle or cluster of paths, resulting from surface roughness or irregularities. The random gain of the bundle is called multipath fading [7], [8], [9], [10].

\subsection{Types of Smart Antenna Systems}

Terms commonly heard today that embrace various aspects of a smart antenna system technology include intelligent antennas, phased array, SDMA, spatial processing, digital beam forming, adaptive antenna systems, and others. Smart antenna systems are customarily categorized, however, as either switched beam or adaptive array systems.

The following are distinctions between the two major categories of smart antennas regarding the choices in transmit strategy:

- Switched Beam - a finite number of fixed, predefined patterns or combining strategies (sectors)

- Adaptive Array—an infinite number of patterns (scenario-based) that are adjusted in real time

\subsubsection{Switched Beam Antenna}

Switched beam antenna systems form multiple fixed beams with heightened sensitivity in particular directions. These antenna systems detect signal strength, choose from one of several predetermined, fixed beams, and switch from one beam to another as the mobile moves throughout the sector.

Instead of shaping the directional antenna pattern with the metallic properties and physical design of a single element (like a sectorized antenna), switched beam systems combine the outputs of multiple antennas in such a way as to form finely sectorized (directional) beams with more spatial selectivity than can be achieved with conventional, single-element approaches fig (2).
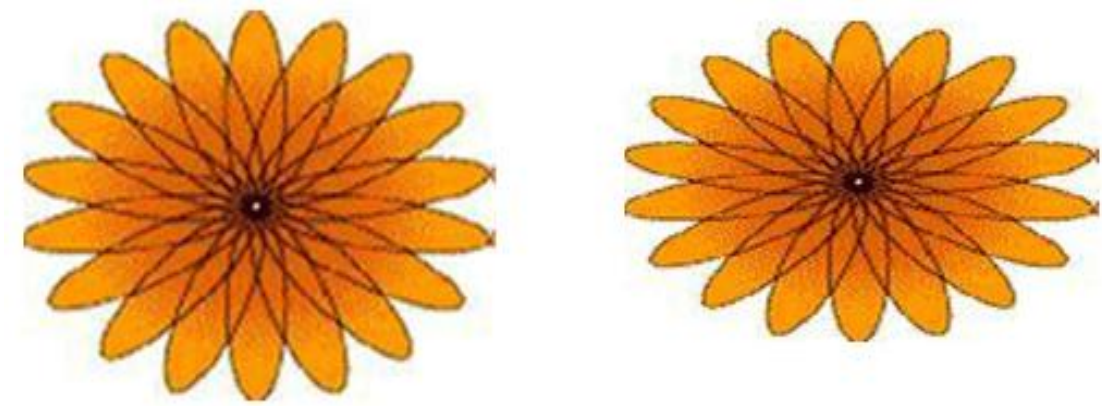

Fig. 2: Switched Beam System Coverage Patterns (Sectors)

\subsubsection{Adaptive Array Antenna:}

Adaptive antenna technology represents the most advanced smart antenna approach to date. Using a variety of new signal-processing algorithms, the adaptive system takes advantage of its ability to effectively locate and track various types of signals to dynamically minimize interference and maximize intended signal reception. 


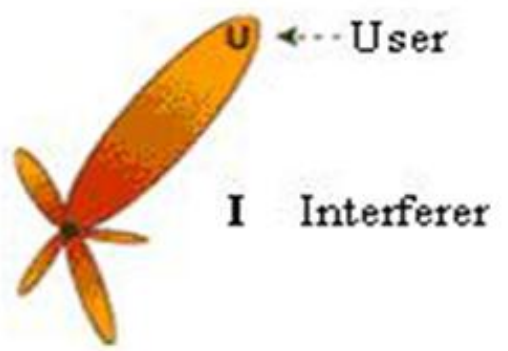

Fig. 3: Adaptive Array Antenna

Both systems attempt to increase gain according to the location of the user; however, only the adaptive system provides optimal gain while simultaneously identifying, tracking, and minimizing interfering signals.

Adaptive Array Coverage: A representative depiction of a main lobe extending toward a tser with a null directed toward a co-channel interferer as shown in fig (3).

\section{The Goals of a Smart Antenna System}

The dual purpose of a smart antenna system is to augment the signal quality of the radio-based system through more focused transmission of radio signals while enhancing capacity through increased frequency reuse.

Table 1: Features and Benefits of Smart Antenna Systems [12].

\begin{tabular}{|c|c|}
\hline Feature & Benefit \\
\hline $\begin{array}{l}\begin{array}{l}\text { Signal } \\
\text { antennas } \\
\text { are }\end{array} \begin{array}{l}\text { combined to } \\
\text { optimize }\end{array} \\
\text { available power required to establish } \\
\text { given level of coverage. }\end{array}$ & $\begin{array}{l}\text { Better Range / Coverage-Focusing the energy sent out into the cell } \\
\text { increases base station range and coverage. Lower power requirements } \\
\text { also enable a greater battery life and smaller/lighter handset size. }\end{array}$ \\
\hline $\begin{array}{l}\text { Interference Rejection-Antenna } \\
\text { pattern can be generated toward } \\
\text { cochannel interference sources, } \\
\text { improving the signal-to-interference } \\
\text { ratio of the received signals. }\end{array}$ & $\begin{array}{l}\text { Increased Capacity-Precise control of signal nulls quality and } \\
\text { mitigation of interference combine to frequency reuse reduce distance } \\
\text { (or cluster size), improving capacity. Certain adaptive technologies } \\
\text { (such as space division multiple access) support the reuse of } \\
\text { frequencies within the same cell. }\end{array}$ \\
\hline $\begin{array}{l}\text { Spatial Diversity-Composite } \\
\text { information from the array is used to } \\
\text { minimize fading and other undesirable } \\
\text { effects of multipath propagation. }\end{array}$ & $\begin{array}{l}\text { Multipath Rejection-can reduce the effective delay spread of the } \\
\text { channel, allowing higher bit rates to be supported without the use of } \\
\text { an equalizer, improved bit error rate (due to decreased amount of } \\
\text { multipath and ISI). }\end{array}$ \\
\hline $\begin{array}{l}\text { SDMA-SDMA continually adapts to } \\
\text { the radio environment through } \\
\text { intelligent / smart antenna. }\end{array}$ & $\begin{array}{l}\text { Providing each user with uplink and downlink signals of the highest } \\
\text { possible quality and can adapt the frequency allocation to where the } \\
\text { most users are located. }\end{array}$ \\
\hline $\begin{array}{l}\text { Power Efficiency-combines the inputs } \\
\text { to multiple elements to optimize } \\
\text { available processing gain in the } \\
\text { downlink (toward the user) }\end{array}$ & $\begin{array}{l}\text { Reduced Expense-Lower amplifier costs, power consumption, and } \\
\text { higher reliability will result. Lower power consumption reduces not } \\
\text { only interferences but also reduces RF pollution (ease health hazard). } \\
\text { It will also result in reduction of scares energy resource (diesel } \\
\text { consumption) and save foreign currency. }\end{array}$ \\
\hline
\end{tabular}

\section{Application In Mobile Communications}

A space-time processor (smart 'antenna') is capable of forming transmit/receive beams towards the mobileof interest. At the same time it is possible to place spatial nulls in the direction of unwanted interferences. This capability can be used to improve the performance of a mobile communication system.

\subsection{Increased Antenna Gain}

The 'smart' antenna forms transmit and receive beam s. Therefore, the 'smart' antenna has a higher gain than a conventional omni-directional antenna. The higher gain can be used to either increase the effective coverage, or to increase the receiver sensitivity, which in turn can be exploited to reduce transmit power and electromagnetic Radiation in the network [2].

\subsection{Decreased inter symbol interference}

Multipath propagation in mobile radio environments leads to ISI. Using transmit and receive beams that are directed towards the mobile of interest reduces the amount of Multipath and ISI. Figure 5 shows the interference reduction. 


\section{Application in Mobile Communications}

A space-time processor (smart 'antenna') is capable of forming transmit/receive beams towards the mobileof interest. At the same time it is possible to place spatial nulls in the direction of unwanted interferences. This capability can be used to improve the performance of a mobile communication system.

\subsection{Increased Antenna Gain}

The 'smart' antenna forms transmit and receive beam s. Therefore, the 'smart' antenna has a higher gain than a conventional omni-directional antenna. The higher gain can be used to either increase the effective coverage, or to increase the receiver sensitivity, which in turn can be exploited to reduce transmit power and electromagnetic Radiation in the network [2].

\subsection{Decreased inter symbol interference}

Multipath propagation in mobile radio environments leads to ISI. Using transmit and receive beams that are directed towards the mobile of interest reduces the amount of Multipath and ISI. Figure 5 shows the interference reduction.

\section{Conclusion}

In conclusion to this paper "Smart Antenna" systems are the antennas with intelligence and the radiation pattern can be varied without being mechanically changed. With appropriate adaptive algorithms such as Recursive Least Square Algorithm (RLS) the beam forming can be obtained. As the system uses a DSP processor the signals can be processed digitally and the performance with a high data rate transmission and good reduction of mutual signal interference. The narrow beams get rid of interference, allowing many users to be connected with in the same cell at the same time using the same frequencies and can adapt the frequency allocation to where the most users are located. With adaptive beam forming, spectral efficiency of the cell could be multiplied at leastten times [6]. Smart antennas effectively reduce the power consumption which in turn avoids RF pollution, minimize health hazard and save scarce resource (diesel \& foreign exchange). Indeed it has been argued that performance requirement of a future cellular communication system cannot be made without the use of smart antennas.

\section{References}

[1] Dr. Mir Mohammad Azad \& Abu HasnatShohel Ahmed "Development of smart antenna for future generation wireless internet connection" IJCSNS international journal of computer science and network security, vol. 10, no. 10, october 2010.

[2] RameshwarKawitkar\& D G Wakde "Advances in smart antenna system" journal of scientific \& industrial research, vol. 64, september 2005, pp 660-665.

[3] AgiusAA. Leach S M. Suvannapattana P, Lund T \& Saunders S R "Intelligent Handheld Antennas for Mobile Communications Beyond the 2nd Generation" version 2.0.2, p12.

[4] Trent K, Are Smart Antennas the way to Non-Line-of-Sight? http://www.shorecliffcommunications.com/magazine/volume.asp (2001).

[5] Litwa J, Digital Beam forming in wireless communications, 1996.

[6] Steyskal H, Digital Beam forming antennas, An introduction, Microwave J, 30 (1987) 107-124.

[7] Martin Cooper, Marc Goldburg, "Intelligent Antennas: Spatial Division Multiple Access" Annual Review of Communications, 1996

[8] Joseph Shapira, "Microcell Engineering in CDMA cellular Networks" IEEE Transactions on Vehicular Technology, Vol 43, No. 4, Nov 1994.

[9] RameshwarKawitkar, "Issues in Deploying Smart Antennas in Mobile Radio Networks " proceedings of World Academy of Science, Engineering and Technology vol 31 july 2008, ISSN 2070-3740.

[10] Ch. Santhi Rani, Dr. P V Subbaiah, Dr. K Chennakesavareddy, "Smart Antenna Algorithms for WCDMA Mobile Communication Systems" IJCSNS International Journal of Computer Science and Network Security, vol 8No. 7, July 2008.

[11] Na Yao," A CBR Approach for Radiation Pattern Control in WCDMA Networks" submitted for the degree of doctor of philosophy, Department of Electronic Engineering Queen Mary, University of London, January 2007.

[12] Minsoo Kim, SungsooAhn, Seungwon Choi and Tapan K. Sarkar, "An Adaptive Beam-forming Algorithm for Smart Antenna System in Practical CDMA Environments" IEICE Trans. Commun. Vol. E86-B, No. 3, March 2003.

[13] Ayman F. Naguib, ArogyaswamiPaulraj and Thomas Kailath, "Capacity Improvement with Base-Station Antenna Arrays in Cellular CDMA" IEEE Transactions on Vehicular Technology, Vol. 43, No. 3, August 1994.

[14] Magnus Madfors, Kenneth Wallstedt, Sverker Magnusson, HakanOlofsson, Per-Ola Backman, and Stefan Engstrom, "High Capacity with Limited Spectrum in Cellular Systems” IEEE Communications Magazine, pp 38-45, August 1997.

[15] TiongSiehKiong, Mahamod Ismail and Azmi Hassan, "WCDMA Forward Link Capacity Improvement by Using Adaptive Antenna with Genetic Algorithm Assisted MDPC Beamforming Technique” Journal of Applied Sciences 6 (8): 1766-1773, 2006 ISSN $1812-5654$. 